\title{
Spray droplet characterization measurements using global rainbow refractometry and microscope analysis
}

\author{
Pan $\mathrm{Qi}^{1, \mathrm{a}^{*}}$, Yue bangshan ${ }^{1, \mathrm{~b}}$ and Zhang Zijia ${ }^{1, \mathrm{c}}$ \\ ${ }^{1}$ School of information and control \& CICAEET, Nanjing University of information science \& \\ technology, Nanjing, 210044, China \\ aqpan@nuist.edu.cn, byuebangshan717@163.com, 'zhzijia@126.com
}

Keywords: Spray, droplet diameter, measurement, rainbow refractometry, microscope

\begin{abstract}
The characterization of spray behavior especially the droplets diameter distribution plays an important role in many applications. In this paper, the droplets diameter distributions of a flat fan water spray were measured using both the Global Rainbow Refractometry (GRR) method and sampling method with microscope analysis. a rainbow parameter inversion algorithm is proposed and applied to the GRR measurement, which based on regularized NNLS and regularization method. The sampling droplets were detected using a microscope, and the droplets images were transformed and rebuilt by several image processing techniques. The experiment results from both methods were compared and the errors were analyzed.
\end{abstract}

\section{Introduction}

Spray droplets parameters measurements especially the diameter distribution detecting are very attractive in many fields, as the droplets diameter distribution can give a good knowledge to reveal and analyze the nozzle atomization, which is widely used in industrial applications, medical productions, agriculture planting, fire protection and so on. Nowadays, various kinds of measurement techniques for spray droplets diameter measurement have been proposed [1-3].

Among these methods, the Global Rainbow Refractometry (GRR) [4], which is based on the detection of a monochromatic rainbow interference pattern generated by spray droplets that are illuminated by a laser beam, is widely studied, as it not only can give the droplets diameter distribution but also the refractive index of the droplets, which has an almost linear relationship with droplets temperature and concentration, and can give thermal and chemical properties of the droplets.

The main signal of the global rainbow is its main bow, which is always captured by a CCD camera. Former research has revealed that the main bow of global rainbow pattern is mostly determined by both the mean diameter and refractive index of the particles, and mathematic model to get the two parameters is a typical inverse problem, so the accuracy of this method very much depends on the inverse algorithm. Also the accuracy of GRR is multiple affected by droplets nonsphericity [6], ripple structure and noise, so a contrastive method is needed to evaluate the error of GRR method.

In this paper, a GRR inversion algorithm is proposed, which was applied on an experimental measurements performed on a flat fan atomizer nozzle. A sampling method was chosen as a contrastive measurement, and the sampling droplets images captured by a microscope were analyzed using image processing technique. The results from two methods were compared and the errors of both methods were analyzed. The structure of the paper is as follows: Firstly, an inversion algorithm is proposed. Secondly, the experiments on spray droplets of flat fan nozzle is done using both GRR and sampling methods. Lastly, the results from both methods were compared and the errors were analyzed.

\section{Inverse algorithm}

The light intensity distribution of the global rainbow can be discretized by a sum using numerical quadrature, which is as follows: 


$$
\left[\begin{array}{c}
I\left(\theta_{1}\right) \\
I\left(\theta_{2}\right) \\
\vdots \\
I\left(\theta_{n}\right)
\end{array}\right]=A f=A\left[\begin{array}{c}
f\left(d_{1}\right) \\
f\left(d_{2}\right) \\
\vdots \\
f\left(d_{i}\right)
\end{array}\right]=\left[\begin{array}{l}
a_{11}, a_{12}, \cdots a_{1 i} \\
a_{21}, a_{22}, \cdots a_{2 i} \\
\vdots \\
a_{n 1}, a_{n 2}, \cdots a_{n i}
\end{array}\right]\left[\begin{array}{c}
f\left(d_{1}\right) \\
f\left(d_{2}\right) \\
\vdots \\
f\left(d_{i}\right)
\end{array}\right]
$$

Where $\mathrm{I}\left(\theta_{\mathrm{n}}\right)$ represents the light intensity measured at the nth scattering angle position, $\mathrm{A}$ is a matrix and the item $a_{n j}$ describes the light intensity of the droplet with diameter value of $d_{i}$ at the nth scattering angle position, and the vector $f$ is the diameter distribution. The inversion process is using the measured light intensity $\mathrm{I}\left(\theta_{\mathrm{n}}\right)$ and the computed matrix $\mathrm{A}$ to get the diameter distribution $\mathrm{f}$. It is a typical inversion problem of a first-kind Freholm integral equation. In order to avoid ill-conditioned problem and the multiple solutions, an inversion algorithm based on Non-Negative Least-Squares (NNLS) method is presented, and in order to get a one only smooth solution, the regularization method is also concerned. So the inversion of the first-kind Freholm integral equation changes into the equation shown as follows:

$$
f=\min _{f}(\|A f-I\|+\gamma\|H f\|), \quad \mathrm{f} \geq 0
$$

Where $\gamma$ is the regularization parameter determined by L-curve method. $\mathrm{H}$ is the Tikhonov regularization matrix. The original value of the refractive index and diameter distribution can be estimated from the angle position and main bow width of the global rainbow [4-5].When the original diameter distribution is determined, a recomputed global rainbow signal can be built. Comparing with the measured signal, the refractive index adjusting can be made according to the bias of the main peak position, as it is found that with the increase of refractive index, the whole rainbow pattern is moved towards the large angle direction, but the angular inter-fringe structure changes little. The diameter range is also recalculated according to the new mean diameter from the inversed distribution. All the processes are repeated to minimize the bias between the measured signal and the recomputed global rainbow, and the best value of refractive index and diameter distribution will be obtained. The whole flowchart of the algorithm is shown in Fig.1.

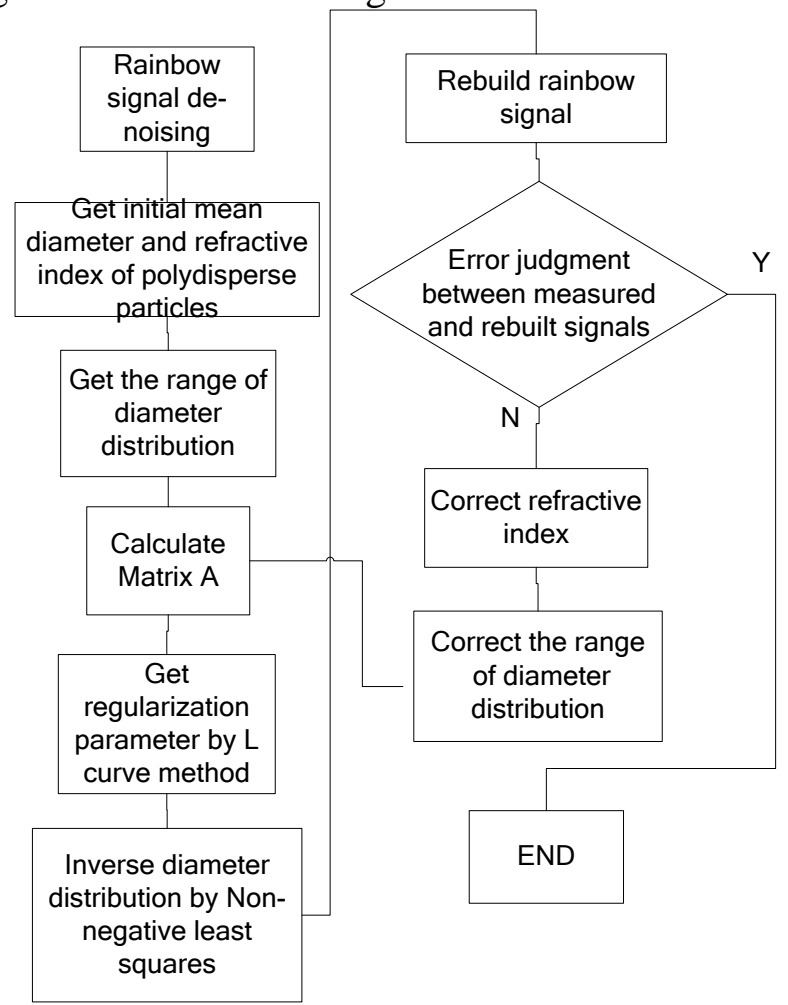

Fig.1 Flowchart of inversion algorithm 


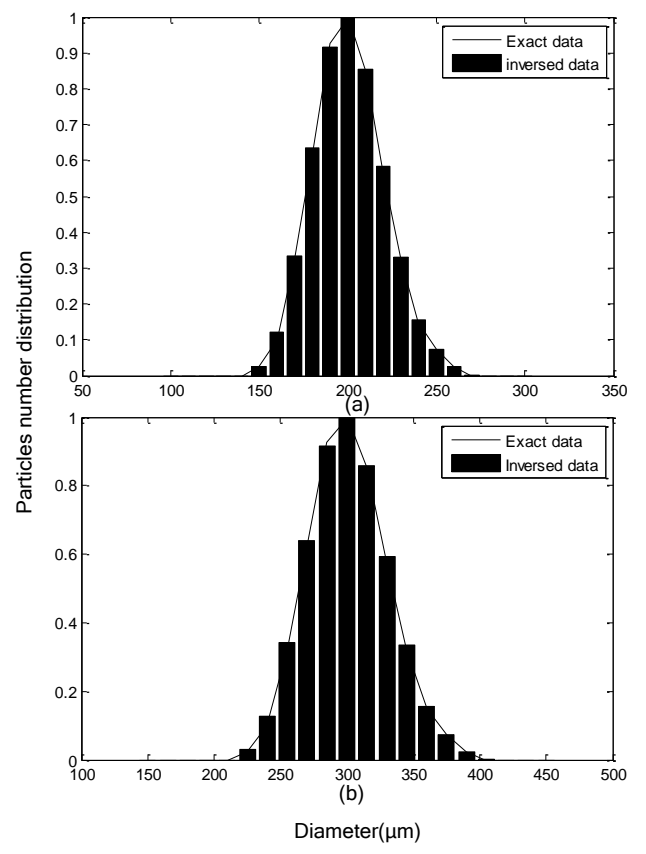

Fig. 2 Inversion results for a log-normal diameter distribution with a refractive index of 1.335. (a) Mean diameter is $200 \mu \mathrm{m}$, the dispersion coefficient is 0.1 ; (b) Mean diameter is $300 \mu \mathrm{m}$, the dispersion coefficient is 0.1 .

Two different log-normal diameter distributions are chosen to test the ability of the inversion algorithm, with the chosen refractive index is 1.335 . The measured global rainbow is simulated by Mie theory. The inversed diameter distributions are shown in Fig.2, and the inversed refractive index is 1.335.The inversion results showed a very good agreement with the input values, both for the diameter distribution and for the refractive index.

\section{Experiment}

In order to measure the droplets diameter distribution and refractive index of spray, experiments were carried out using water atomization system. The experimental apparatus is sketched in Fig 3. The spray is generated by a flat fan atomizer nozzle. The optical system for measurement is also shown in Fig 3 . The laser power is $100 \mathrm{~mW}$. The rainbow light formed by the scattering droplets is collected by a lens with large diameter and is finally received by a linear CCD placed in the image plane of the lens system. An adjustable mirror is used to change the angle of the laser beam, with the polarization direction and the intensity of the incident light regulated by polarizer1 and polarizer 2 respectively, and a spatial filter selects the probe volume. The measurement is taken at distance $\mathrm{z}=$ $20 \mathrm{~cm}$ under the nozzle Introduction at the ambient temperature of $30^{\circ} \mathrm{C}$. A small plate filled with silicon oil was fixed on a slide platform which was driven by a step motor. When the experiments began, firstly the global rainbow measurement system received the scattered rainbow signal from the measuring region. Then the small plate passed through the measuring area at a uniform speed collecting droplets. As both the silicon oil and the water have the similar density, the collected droplets suspended keeping the shapes almost spherical. Lastly the plate with collected droplets was detected using a microscope, and a video record was made during the microscope scanning process.

Images from different regions of the plate were extracted from the video, and all the images were transformed into grayscale images. A homomorphic filtering technique is applied to remove the uneven illumination effects. After the edges of the droplets in the images is enhanced by Laplace method, all the images transformed into binary images using adaptive thresholding. Several steps were done to solve the inside small hole and multiple edges problems, then after Hough transforming the shapes of the droplets were rebuilt. Then the droplets diameters were determined after the calibrating the relationship between length and pixel numbers. So the diameter distributions were got 
after adding up the diameters calculated from all the images. The flowchart of microscope image analysis is shown in Fig.4, and an example of image transforming results is given in Fig.5.

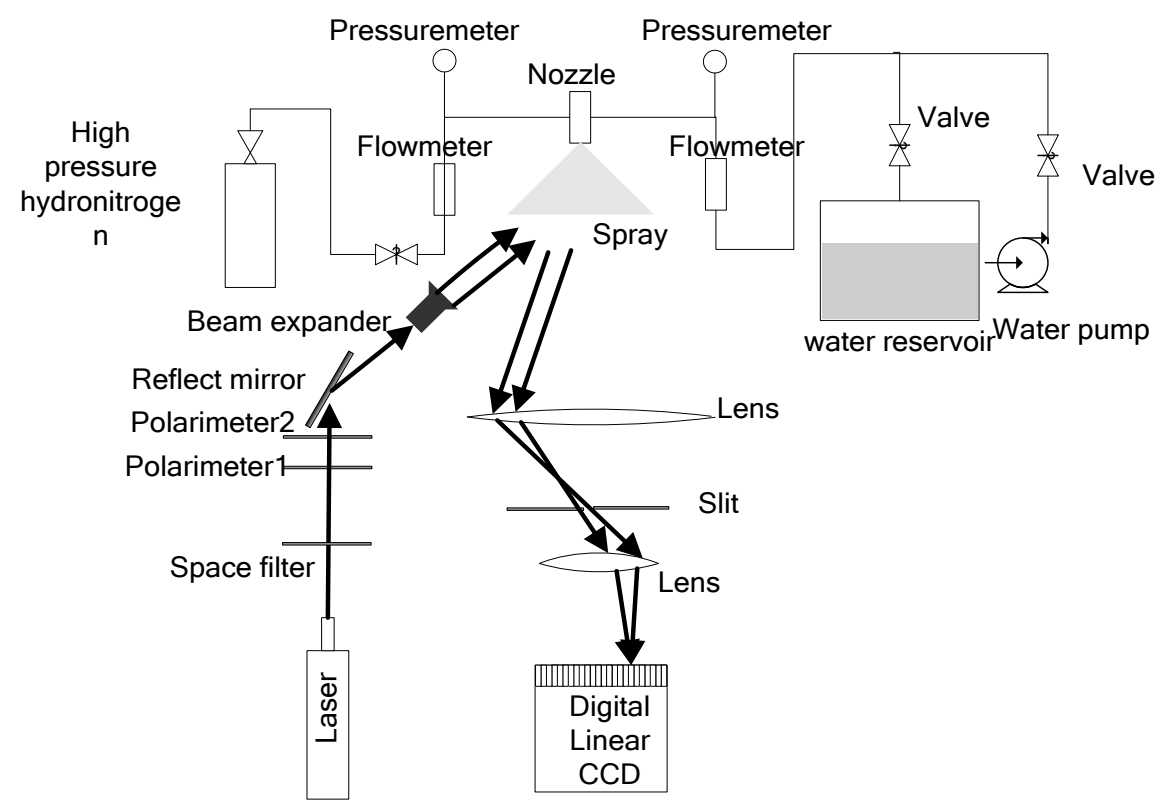

Fig.3 Sketch of experimental setup
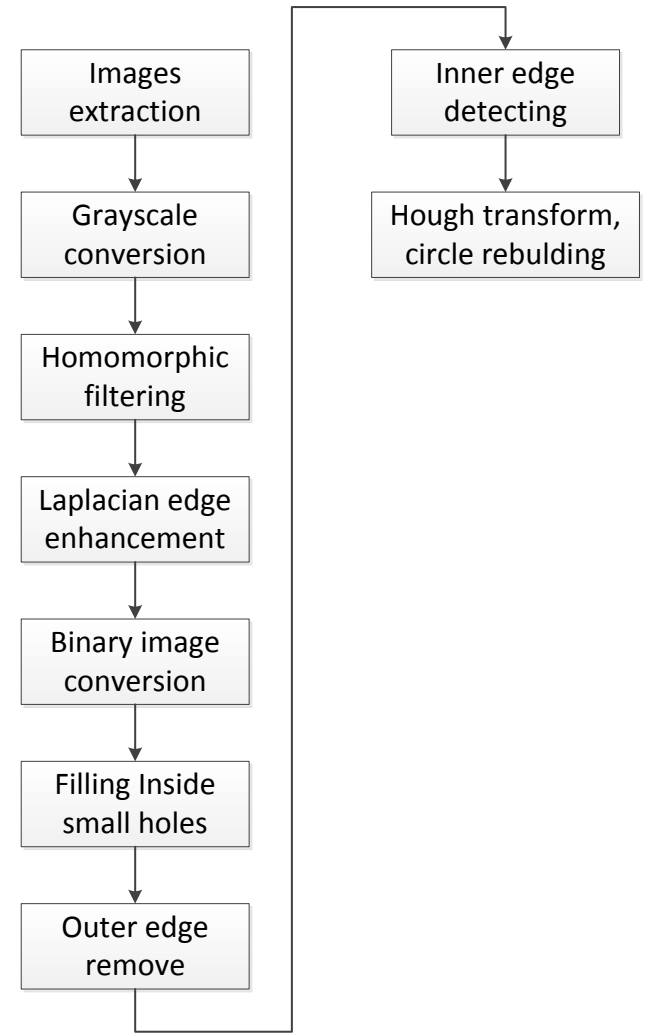

Fig.4 Flowchart of microscope image analysis
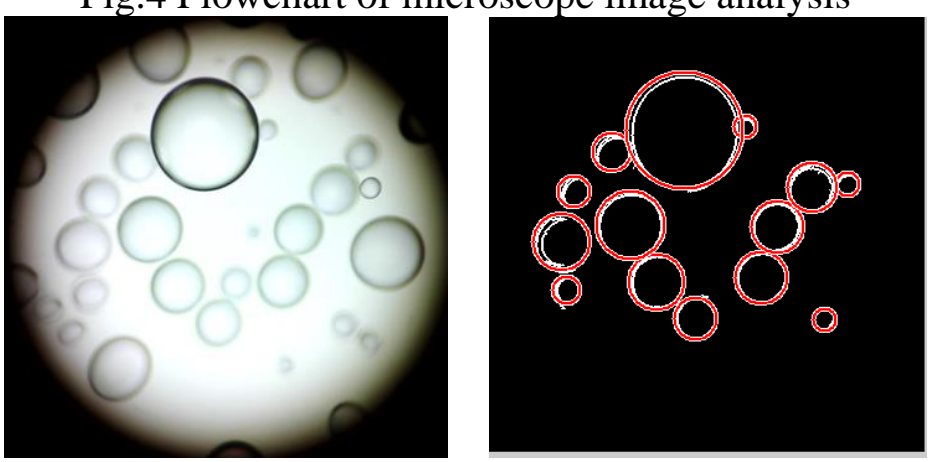
(a)

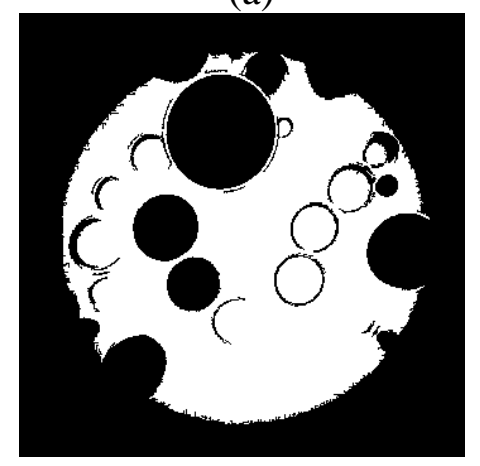

(b)

(c)

Fig.5 Image transforming results. (a) Original image;(b) Binary image;(c) Rebuilding image

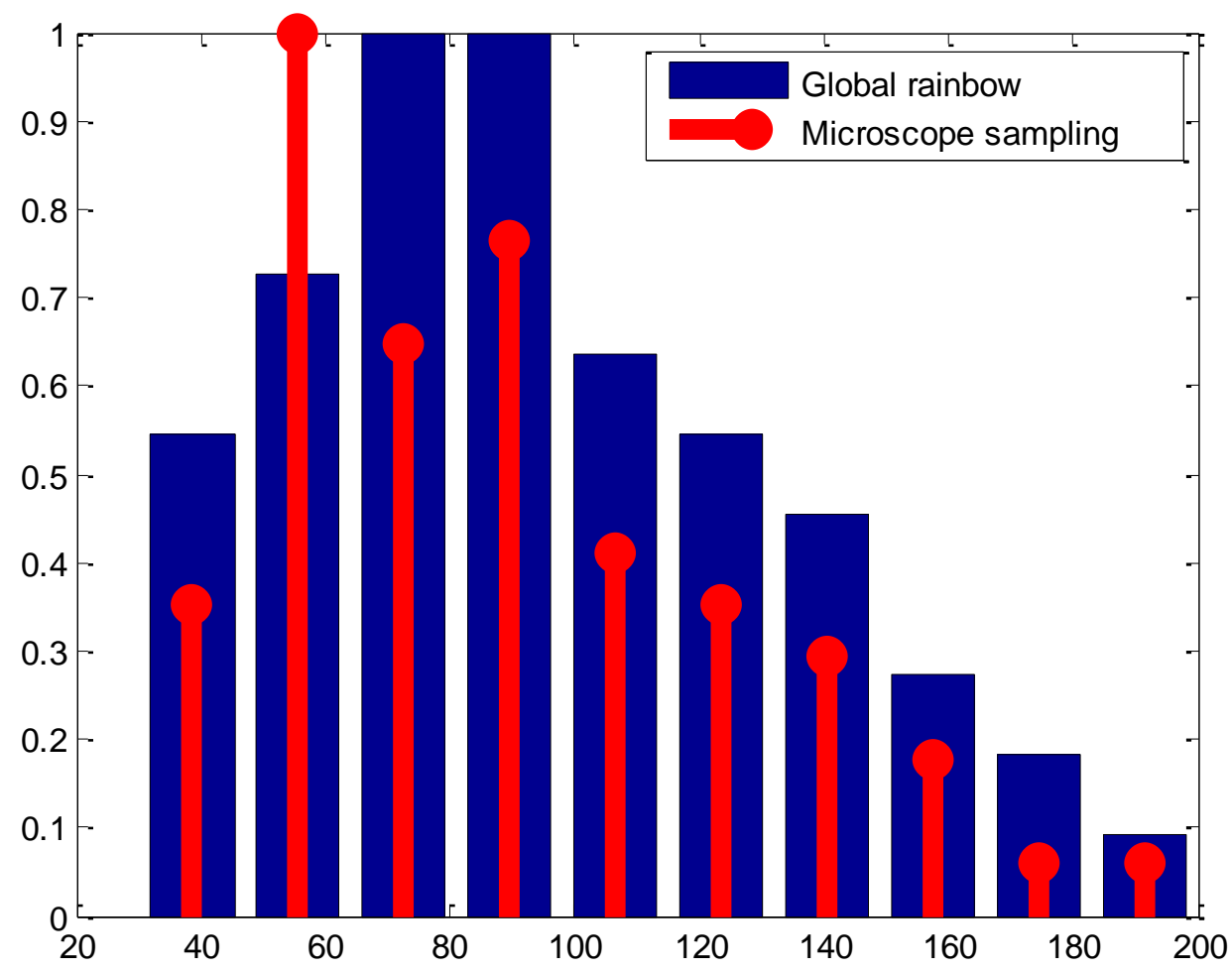

Fig.6 Experimental results from two methods

The measured refractive index value by GRR is 1.332 , and the diameter distributions measured from GRR and sampling method is shown in Fig.6. The diameter distributions from two methods are normalized separately. It is shown that the diameter distribution from GRR has one main peak which is more consistent with the actual situation. The distribution from sampling method has two peak values, and the peak value around $50 \mu \mathrm{m}$ is especially large comparing to the result from GRR. It was probably caused by the sputtering small droplets collected during the sampling process. As the sampling method is an intrusive measurement, though the sampling process was quick and stable the small droplets created from spray droplets hitting the sampling plate were unavoidable. In spite of this, the rest of the distributions from two methods have the same trend, and the value differences are mainly because of the normalization, which indicates the validity and accuracy of the GRR method.

\section{Conclusion}

In this paper, the droplets diameter distributions of a flat fan water spray were measured using both the Global Rainbow Refractometry (GRR) method and sampling method with microscope analysis. A rainbow parameter inversion algorithm is proposed and applied to the GRR measurement, which 
based on regularized NNLS and regularization method. The sampling droplets were detected using a microscope, and the droplets images were transformed and rebuilt by several image processing techniques. The experimental results from both methods were compared and the errors were analyzed.

\section{Acknowledgement}

This research (Project No. 51206082) is supported by National Science Foundation of China.

\section{References}

[1] Long Wuqiang, Ohtsuka, Hiroyuki, Obokata, et al ,"Characterization of conical spray flow for diesel engine by means of laser Doppler methods," Transactions of the Japansociety of Mechanical Engineers 60(576), (1994) 2917-2923.

[2] A.R.Glover, S.M.Skippon, R.D.Boyle, "Interferometric laser imaging for droplet sizing: a method for droplet-size measurement in sparse spray systems," Applied Optics 34(8), (1995) 8409-8421.

[3] Jos Pastor, Jos Lpez, J Juli, et al,"Planar Laser-Induced Fluorescence fuel concentration measurements in isothermal Diesel sprays," Applied optics 10(7), (2002) 303-323.

[4] P A J van Beeck, D Giannoulis, L Zimmer, et al, "Global rainbow thermometry for droplet temperature measurement," Optics Letters 24(23), (1999) 1696-1698.

[5] Pan Qi, "Characterization of a flat fan air-blast atomizer spray by global rainbow refractometry", SPIE proceedings, (2011)

[6] Pan Qi," Experiment validation of non-sphericity effect on monochromatic rainbow measurement ", SPIE proceedings, (2012) 